\title{
Una estrategia para la documentación de lecciones aprendidas y buenas prácticas en empresa de desarrollo de software
}

\author{
Becerril-Sitten Juan Pablo ${ }^{1}$, Perez-Soltero Alonso ${ }^{1 *}$, Barcelo-Valenzuela Mario ${ }^{1}$, Sanchez-Schmitz Gerardo , \\ Cirett Galan Federico ${ }^{1}$
}

${ }^{1}$ Universidad de Sonora. Departamento de Ingeniería Industrial.

\begin{abstract}
Resumen
Muchas organizaciones para mejorar sus servicios o productos han optado por herramientas que les brinden una ventaja competitiva o que les permitan realizar sus procesos más eficientemente. Una de las herramientas que han tomado importancia en las organizaciones es el registro de buenas prácticas o lecciones aprendidas, esto con el beneficio de que aprendan de experiencias pasadas posibilitando la creación de nuevo conocimiento útil en nuevos métodos o procesos. Ante la necesidad de mejora que tienen las organizaciones, el objetivo de este trabajo es proponer una estrategia que permita facilitar la captura de lecciones aprendidas y buenas prácticas en una empresa de desarrollo de software. Debido a la situación existente en la organización de estudio, se desarrolló e implementó una metodología que comprendiera identificar a expertos en los procesos organizativos; diseñar una estructura que facilite la captura de las lecciones aprendidas y buenas prácticas; e implementar la estructura diseñada en los empleados de la organización. Como principal resultado, se obtuvo el registro de éxitos, fracasos o experiencias que permitieran la creación de buenas prácticas y lecciones aprendidas, siendo de utilidad para la mejora de las actividades laborales dentro la organización de estudio.
\end{abstract}

Palabras Clave: buenas prácticas, lecciones aprendidas, conocimiento, documentación.

\section{A strategy for documenting lessons learned and good practices in a software development company}

\begin{abstract}
Many organizations have opted for tools that offer them a competitive advantage or allow them to carry out their processes more efficiently to improve their services or products. One of the tools that have taken importance in organizations is the documentation of good practices or lessons learned, this with the benefit of learning from past experiences enabling the creation of new useful knowledge in new methods or processes. Given the need for improvement that organizations have, the objective of this paper is to propose a strategy that facilitates the capture of lessons learned and good practices in a software development company. Due to the situation existing in the study organization, a methodology was developed and implemented that included identifying experts in the organizational processes; design a structure that facilitates the capture of lessons learned and good practices; and implement the structure designed in the employees of the organization. As a main result, the record of successes, failures or experiences that allowed the creation of good practices and lessons learned was obtained, being useful for the improvement of work activities within the study organization.
\end{abstract}

Keywords: good practices, lessons learned, knowledge, documentation.

*Autor para envío de correspondencia: Universidad de Sonora. Departamento de Ingeniería Industrial. Blvd. Luis Encinas y Rosales S/N, Col. Centro, Hermosillo, Sonora, México, C.P. 83000. Tel/Fax: (662) 259-21-60. E-mail: aperez@industrial.uson.mx

(c) 2019 Editorial UNISON — URN. Derechos reservados. 


\section{INTRODUCCIÓN}

El conocimiento es uno de los activos más importantes para las organizaciones debido a que aporta valor a los productos o servicios que estas ofrecen (Hernández, 2014). Puede definirse como aquel conjunto de información o percepciones almacenadas mediante la experiencia, aprendizaje u observación (Wiig, 1993; Pérez, 2008).

Es de importancia que en una empresa se considere el conocimiento como una ventaja competitiva, por el hecho que permite el desarrollo de la innovación de nuevos métodos o estrategias de negocio que faciliten sobresalir y superar a sus competidores (Nonaka, 1991; Davenport y Prusak, 1998).

Para lograr que el conocimiento sea útil, este debe ser formalizado y sistematizado, ya sea en datos o procedimientos codificados; también transmitido de un individuo a otro (Nonaka y Takeuchi, 1995).

La gestión del conocimiento (GC) es un campo multidisciplinario, que tiene como principal objetivo el hacer disponible el conocimiento a un conjunto de personas (Maier, 2007; Sánchez, 2016). A partir de la creación, captura y diseminación del conocimiento se es capaz de generar nuevos conocimientos, basados en experiencias positivas o negativas de aquello que funcionó o no en el pasado (Grey, 1996; Rigby, 2009; Dalkir, 2011).

Todo aquello que hace referencia a experiencias que funcionaron y que han obtenido resultados esperados o benéficos, es denominado como buenas prácticas o mejores prácticas (Muntaner y col., 2016). Trabajar con buenas prácticas, supone identificar, diseminar y compartir conocimientos y experiencias exitosas, contrastadas con el logro de objetivos; además, estas deben ser innovadoras, efectivas, replicables y que sirvan como modelo para desarrollar iniciativas en otros lugares (Santiso, 2002; FAO, 2015).

Por otra parte, las lecciones aprendidas son los conocimientos apropiados sobre algún proceso o experiencia, a partir de la reflexión y el análisis crítico de sus resultados (BID, 2011). A través de las lecciones aprendidas se es capaz de identificar los aspectos positivos y/o negativos adquiridos por experiencias, siendo útiles para identificar: factores de éxito; deficiencias en distintos métodos, estrategias, procesos o proyectos; y soluciones potenciales a problemas recurrentes (Weber y col., 2001; BID, 2011).

Tan y col. (2006) y Milton (2010) propusieron en sus estudios una plantilla para la captura y re-uso del conocimiento generado en la ejecución de diversos proyectos. En ambas plantillas, en su estructura contemplaban el registro del contexto y descripción del proyecto, causas del origen del éxito o fracaso y acciones recomendables a realizar; esto con la finalidad de identificar lecciones aprendidas originadas en el desarrollo de algún proyecto.

La gestión y documentación de procesos juega un papel importante en la gestión estratégica de las políticas, métodos y prácticas que coordina y utiliza una organización (Bruch y Bellgran, 2013). La ejecución correcta de los procesos dentro de una organización está ligada a la documentación existente y al conocimiento que los empleados poseen (James, 2017).

Cuando no existe un registro de los procesos o prácticas que una organización normalmente realiza, se tiende a que el conocimiento sea extraviado o que la ejecución de distintas actividades se realice con errores (Lucid Software, 2018). Por consiguiente, es valioso que todo conocimiento o experiencia, ya sea en forma de buena práctica o lección aprendida, sea almacenado en alguna herramienta tecnológica que facilite la captura y diseminación del conocimiento de todos los integrantes en una organización (Van Wart y col., 2017; Blurton, 2002).

La documentación de buenas prácticas y lecciones aprendidas puede ser vista en varias empresas de desarrollo de software, debido a que se motiva a los empleados para que ejerzan buenas prácticas en procesos de documentación de código, buena comunicación entre los integrantes de un equipo, definición de metas alcanzables y requerimientos ligados al logro de objetivos, entre otras prácticas más (Visser, 2017; UNS, 2017).

De lo anterior, el objetivo del presente trabajo es describir una estrategia que permita documentar las buenas prácticas y lecciones aprendidas que puedan obtenerse en alguna organización; esto con la finalidad de demostrar la importancia y utilidad que estas tienen para hacer más eficiente los procesos y actividades laborales.

Además de hacer uso o implementar la estrategia en una organización, con el motivo de identificar aquellos procesos que pueden mejorarse o de aquellas actividades que deben replicarse para que la organización desempeñe correctamente sus labores. Esto también con el propósito de verificar si la estrategia propuesta es viable para que otras organizaciones hagan uso de la misma.

\section{MÉTODO}

El presente estudió contempló la elaboración de una metodología que hiciera uso de herramientas de GC para la documentación de buenas prácticas y lecciones aprendidas en una empresa de desarrollo de software. 
AD Sistemas Soluciones en TI es una PyME que tiene como principal actividad laboral el manejo, adaptación y distribución de su sistema ERP. Debido a que enfrenta dificultad al hacer uso de su ERP y de disponer de conocimiento útil para el uso efectivo del mismo dentro de la organización y con sus respectivos clientes; se da a la tarea de aprovechar el conocimiento que reside en los empleados basándose en las funciones rutinarias que realizan en la organización y que son de importancia registrar para que no se presente alguna dificultad al momento de desarrollar o adaptar los módulos del sistema ERP o todo aquello relevante con la capacitación y servicio al cliente. En particular, las áreas de interés para llevar a cabo el presente estudio fueron los módulos de compras y ventas del sistema ERP por ser los más utilizados y en los que más dudas se presentaban durante su desarrollo y/o adaptación.

La investigación tiene un enfoque de carácter descriptivo, con el propósito de recopilar y analizar información existente dentro de la organización de estudio. De acuerdo con la problemática existente en la organización y del análisis de la revisión de la literatura, se parte al diseño de una metodología que considere el uso de estrategias de GC, teniendo como principal punto de interés la obtención o recopilación de buenas prácticas y lecciones aprendidas.

A partir de la revisión de la literatura anteriormente descrita, se identificaron características o fundamentos importantes para el registro de buenas prácticas y lecciones aprendidas, contemplando las siguientes actividades: identificar a expertos; diseñar la estructura para documentar las buenas prácticas y lecciones aprendidas; implementar la estructura propuesta dentro de la organización; y seguimiento, generación y registro de nuevas buenas prácticas y lecciones aprendidas. En la figura 1 se muestra la metodología propuesta.

\section{Identificación de expertos}

Consiste en reconocer aquellas personas que poseen el mayor conocimiento en las funciones primarias de la organización. Esto puede saberse a partir de las responsabilidades que desempeña cada persona y con base en la experiencia y capacidad que estas poseen al realizar sus actividades

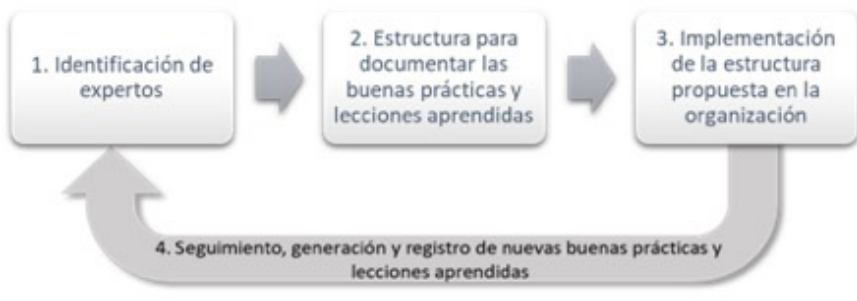

Figura 1. Metodología para la documentación de buenas prácticas y lecciones aprendidas. laborales. Estas son las personas que han experimentado las experiencias y son capaces de identificar las buenas prácticas y lecciones aprendidas que son relevantes de documentar.

\section{Estructura para documentar las buenas prácticas y lecciones aprendidas}

El objetivo de esta actividad es generar una estructura que apoye a la captura de buenas prácticas y lecciones aprendidas. Antes de diseñar esta estructura, es necesario conocer qué información o criterios son de utilidad para el correcto registro de las lecciones aprendidas o buenas prácticas.

Algunos criterios en los cuales basarse para el registro de estas, son los siguientes: objetivo o actividad; contexto; impacto del objetivo o experiencias obtenidas; acciones recomendadas a realizar; área responsable; y lección aprendida o buena práctica (FAO, 2015).

Teniendo en cuenta los criterios anteriores, se parte a la realización de un formato que apoye a la documentación o registro de buenas prácticas y lecciones aprendidas. Una propuesta de cómo documentar las buenas prácticas y lecciones aprendidas se muestra en la tabla 1.

\section{Implementación de la estructura propuesta en la organización}

Al tener ya diseñada la estructura de la documentación, se procede a la captura de las buenas prácticas y lecciones aprendidas. Estas se definen a partir de los criterios establecidos en el formato creado (tabla 1) y a través del apoyo de los empleados clave o con mayor conocimiento en las funciones laborales, con el propósito de que establezcan acciones importantes que aporten valor a los procesos que realiza la organización.

\section{Seguimiento, generación y registro de nuevas buenas prácticas y lecciones aprendidas}

Por último, debe llevarse a cabo un seguimiento de las buenas prácticas y lecciones aprendidas, ya que de esta manera se tendrá control del cumplimiento de estas, la generación y registro de nuevas, y el desarrollo de una manera más eficiente o productiva de las actividades laborales en la organización.

\section{RESULTADOS Y DISCUSIÓN}

Según las actividades definidas anteriormente en la metodología; los resultados obtenidos se presentan a continuación. 
Tabla 1. Estructura para documentar lecciones aprendidas o buenas prácticas. Adaptado de FAO (2015).

\begin{tabular}{|c|c|}
\hline CRITERIO & DESCRIPCIÓN DEL CRITERIO \\
\hline Objetivo o actividad & Nombre del objetivo o actividad. \\
\hline Contexto & Descripción del contexto, circunstancias o razones donde se aplica el objetivo. \\
\hline Impacto del objetivo o experiencias obtenidas & $\begin{array}{l}\text { Descripción de experiencias positivas o negativas generadas al realizar el objetivo o } \\
\text { actividad, así como también la utilidad de este. }\end{array}$ \\
\hline Acciones recomendadas a realizar & Principales acciones para lograr eficientemente el objetivo o actividad. \\
\hline Área responsable & Área encargada de ejercer la buena práctica o lección aprendida. \\
\hline Lección aprendida o buena práctica & $\begin{array}{l}\text { Describir aquello que, a través de la reflexión de la actividad u objetivo realizado, } \\
\text { requiere mejorar, hacer diferente o replicarse para cumplir con éxito el propósito } \\
\text { del objetivo o actividad, para lograr buenos resultados y algún impacto benéfico en } \\
\text { lo realizado. }\end{array}$ \\
\hline
\end{tabular}

\section{Identificación de expertos}

Se identificó que el principal experto en los procesos y herramientas tecnológicas dentro de la organización es el Director General. Esta persona posee grandes conocimientos en el uso del sistema ERP y de las responsabilidades que tiene cada uno de los empleados. También, es la persona que tiene mayor contacto y relación con los clientes, teniendo la capacidad de conocer los requerimientos y necesidades que poseen cada uno de ellos.

\section{Estructura para documentar las buenas prácticas y lecciones aprendidas}

Partiendo de los criterios establecidos en la metodología se realizó la tabla 1, que describe la estructura a definir para hacer registro de las buenas prácticas y lecciones aprendidas. En esta tabla se contemplan los criterios que se consideran más importantes y que facilitan el entendimiento y registro de cada buena práctica o lección aprendida identificada.

\section{Implementación de la estructura propuesta en la organización}

Haciendo uso de la tabla 1 se procedió a capturar las lecciones aprendidas o buenas prácticas que el Director General de la organización y el responsable del trabajo de investigación identificaron dentro de la organización. Para el logro de esto, se monitoreó durante cinco semanas las actividades que realizaban los empleados y mediante la descripción de experiencias pasadas que consideraba importante el Director General, con el motivo de definir acciones que formaran parte de las buenas prácticas o lecciones aprendidas. Entre los puntos que consideraba el director para definir las buenas prácticas o lecciones aprendidas estaban aquellas que brindaran a los desarrolladores mayores conocimientos que les facilitara el mantenimiento de la base de datos y en la actualización y adaptación de los módulos, formatos, tablas o procesos referentes con el propio sistema ERP. Como resultado se documentaron una gran variedad de experiencias que se plasmaron mediante el llenado de la estructura para la definición de buenas prácticas y lecciones aprendidas con datos de la organización. Por ejemplo, la tabla 2 corresponde a la documentación de los módulos del ERP, la tabla 3 pertenece al uso del manual del sistema ERP, mientras que la tabla 4 corresponde con el desarrollo y mantenimiento eficiente de base de datos.

\section{Seguimiento, generación y registro de nuevas buenas prácticas y lecciones aprendidas}

Contemplando el registro inicial de buenas prácticas y lecciones aprendidas, el director de la organización se

Tabla 2. Lecciones aprendidas o buenas prácticas para la documentación de los módulos del sistema ERP.

\begin{tabular}{|c|c|}
\hline CRITERIO & DESCRIPCIÓN DEL CRITERIO \\
\hline Objetivo o actividad & Documentar los módulos del sistema ERP. \\
\hline Contexto & Mantener documentado en un manual los módulos del sistema ERP que maneja la organización. \\
\hline $\begin{array}{l}\text { Impacto del objetivo o experiencias } \\
\text { obtenidas }\end{array}$ & $\begin{array}{l}\text { Permitió conocer información relevante de los módulos documentados, que serán de utilidad para los clien- } \\
\text { tes al momento de hacer uso del sistema ERP. }\end{array}$ \\
\hline Acciones recomendadas a realizar & $\begin{array}{l}\text { Asignar a personal que se encargue de documentar cada módulo. } \\
\text { Tener conocimiento de uso del propio sistema. } \\
\text { Mantener una planeación de documentación, la cual monitoree las modificaciones necesarias a realizar en } \\
\text { el manual. }\end{array}$ \\
\hline Área responsable & Gerencia o atención al cliente. \\
\hline Lección aprendida o buena práctica & $\begin{array}{l}\text { Seguir documentando los módulos para que estos tengan impacto en un futuro en el conocimiento del uso } \\
\text { del sistema ERP por los clientes. } \\
\text { Se conoció cómo deben documentarse los módulos del ERP y qué información deben poseer para que sea } \\
\text { el manual efectivo. } \\
\text { El documentar procesos permite resolver problemáticas referentes con el olvido de uso del sistema. } \\
\text { Hay que mantener la documentación simple y entendible a todo usuario. }\end{array}$ \\
\hline
\end{tabular}


Tabla 3. Lecciones aprendidas o buenas prácticas para el uso del manual del sistema ERP.

\begin{tabular}{|c|c|}
\hline CRITERIO & DESCRIPCIÓN DEL CRITERIO \\
\hline Objetivo o actividad & Uso del manual del sistema ERP. \\
\hline Contexto & Tener conocimiento de las funciones del sistema ERP. \\
\hline $\begin{array}{l}\text { Impacto del objetivo o experiencias } \\
\text { obtenidas }\end{array}$ & $\begin{array}{l}\text { El personal de la organización hace buen uso del sistema ERP, conociendo las funciones esenciales que están } \\
\text { documentadas en el manual. }\end{array}$ \\
\hline Acciones recomendadas a realizar & $\begin{array}{l}\text { Hacer uso del manual cuando sea necesario y se esté en duda al utilizar alguna función del sistema ERP. } \\
\text { El manual debe estar disponible a todo usuario que haga uso del sistema ERP. } \\
\text { Verificar la información que posee el manual regularmente. }\end{array}$ \\
\hline Área responsable & Desarrolladores de software y atención al cliente. \\
\hline Lección aprendida o buena práctica & $\begin{array}{l}\text { Con el uso del manual se creará un ámbito de trabajo eficiente y ayudará a aumentar las competencias perso- } \\
\text { nales de los clientes. } \\
\text { Permitirá al cliente verificar los pasos o recomendaciones a realizar para el uso correcto del sistema. } \\
\text { Se conoció que el personal de la organización tiene dominio de los módulos documentados. }\end{array}$ \\
\hline
\end{tabular}

comprometió a que los empleados realicen las buenas prácticas o lecciones aprendidas que se hayan identificado en diversas actividades o proyectos. También, dado el caso de que se presentasen nuevas buenas prácticas o lecciones aprendidas se tenga el hábito de documentarlas y difundirlas en toda la organización.

En cuanto a la discusión de los resultados, se puede decir que las buenas prácticas obtenidas en cuestión al desarrollo y mantenimiento eficiente de bases de datos, está ligado con ciertas buenas prácticas que establece Visser (2017), por lo cual son buenas prácticas que se han demostrado que han tenido éxito en otras organizaciones.

Por otro lado, la estructura definida para el registro de buenas prácticas y lecciones aprendidas se diseñó a partir del modelo establecido por FAO (2015), el cual contempla características semejantes con las plantillas desarrolladas por Tan y col. (2016) y Milton (2010) en sus respectivas investigaciones.

Tabla 4. Lecciones aprendidas o buenas prácticas para el desarrollo y mantenimiento eficiente de base de datos.

\begin{tabular}{|c|c|}
\hline CRITERIO & DESCRIPCIÓN DEL CRITERIO \\
\hline Objetivo o actividad & Desarrollo y mantenimiento eficiente de base de datos. \\
\hline Contexto & La base de datos del sistema ERP debe estar funcional con el menor número de defectos. \\
\hline $\begin{array}{l}\text { Impacto del objetivo o experiencias } \\
\text { obtenidas }\end{array}$ & $\begin{array}{l}\text { Se ha tenido experiencias en que la programación de cierto código por cierto usuario no es entendible por } \\
\text { otros, siendo necesario que se adecuen a escribir "código limpio". }\end{array}$ \\
\hline Acciones recomendadas a realizar & $\begin{array}{l}\text { Otorgar capacitación a los desarrolladores a través de cursos de lenguaje .NET y SQL. Además de los aspectos } \\
\text { contables que conforma el sistema ERP. } \\
\text { Los desarrolladores utilicen preferentemente el mismo marco de trabajo en el lenguaje de programación uti- } \\
\text { lizado. } \\
\text { Realizar pruebas de software regularmente. }\end{array}$ \\
\hline Área responsable & Desarrolladores de software. \\
\hline Lección aprendida o buena práctica & $\begin{array}{l}\text { Todos los desarrolladores deben poseer conocimiento de cómo es la interrelación entre ciertos módulos. } \\
\text { Tener un código estructurado, donde se tenga estandarizado la nomenclatura de tablas, columnas y procedi- } \\
\text { mientos. } \\
\text { La declaración de variables en la base de datos debe ser consistente en todo código. }\end{array}$ \\
\hline
\end{tabular}

Otra cuestión importante, es el aporte principal que otorga el registro y seguimiento de buenas prácticas y lecciones aprendidas. Esto, puede verse en varios estudios de GC, tal como lo mencionan Maier (2007) y Sánchez (2016), donde demuestran la importancia de la disposición y sistematización del conocimiento; siendo que en este estudio la organización no contaba anteriormente con alguna estrategia relacionada con la GC.

\section{CONCLUSIONES}

Los resultados obtenidos demuestran las buenas prácticas y lecciones aprendidas observadas durante la implementación del estudio, que resultaron útiles definir por el personal de la organización.

Gran parte de las buenas prácticas definidas están ligadas a comprometerse a documentar casos de éxito que pudieran suscitarse con el uso del sistema ERP. También 
de experiencias pasadas que originaron algún aprendizaje en relación con el desarrollo y mantenimiento del sistema ERP, ya sea en la adaptación de módulos o en el control del funcionamiento correcto de la base de datos.

El estudio sirvió para definir una estructura que facilitara la captura de buenas prácticas y lecciones aprendidas, cumpliendo con el objetivo propuesto del trabajo de investigación; además, que pudiera ser de utilidad para que otras organizaciones hagan uso del mismo.

Finalmente, el registro y cumplimiento de las buenas prácticas y lecciones aprendidas es una tarea que la organización debe continuar dándole seguimiento, para aprovechar el conocimiento que pudiera recolectarse de experiencias de procesos importantes que realizan los empleados, esto con la finalidad de minimizar errores y hacer más eficiente el trabajo conjunto de todos los empleados en la organización. Asimismo, esto tendría un impacto benéfico para el cliente por el hecho que las actividades laborales ejercidas por la organización estarían en continua mejora.

\section{AGRADECIMIENTOS}

Al Consejo Nacional de Ciencias y Tecnología (CONACYT) por el apoyo recibido para la realización del proyecto. $\mathbf{0}$,

\section{BIBLIOGRAFÍA}

BID. 2011. Lecciones aprendidas. Recuperado de: https:// publications.iadb.org/bitstream/handle/11319/3855/ Lecciones\%20Aprendidas.pdf?sequence $=1$. Fecha de consulta: 4 de mayo de 2018.

Blurton, C. 2002. New directions of ICT-use in education. Recuperado de: http://www. unesco.org/education/ educprog//wf/dl/edict.pdf. Fecha de consulta: 6 de marzo de 2018.

Bruch, J. y Bellgran, M. 2013. Characteristics affecting management of design information in the production system design process. International Journal of Production Research, 51, 3241-3251.

Dalkir, K. 2011. Knowledge Management in Theory and Practice. 2nd ed. The MIT Press. Cambridge.

Davenport, T. y Prusak L. 1998. Working knowledge. Harvard Business School Press. Boston.

FAO (Food and Agriculture Organization of the United Nations). 2015. Plantilla de buenas prácticas. Recuperado de: http://www.fao.org/3/a-as547s.pdf. Fecha de consulta: 4 de mayo de 2018.

Grey, D. 1996. What is knowledge management? The Knowledge Management Forum. Recuperado de: http:// www.km-forum.org/t000008.htm. Fecha de consulta: 13 de enero de 2017.

Hernández, N. 2014. Teoría de la gestión del conocimiento. Recuperado de: https://www.gestiopolis.com/teoria-de- la-gestion-del-conocimiento/. Fecha de consulta: 6 de marzo de 2018.

James, I. 2017. Process documentation guide. Recuperado de:http://theprocessconsultant.com/processdocumentation//. Fecha de consulta: 6 de marzo de 2018.

Lucid Software. 2018. ¿Qué es la documentación de procesos? Recuperado de: https://www.lucidchart.com/ pages/es/qu\%C3\%A9-es-la-documentaci\%C3\%B3n-de procesos. Fecha de consulta 21 de febrero de 2018.

Maier, R. 2007. Knowledge Management Systems, 3rd ed. Springer. Austria.

Milton, N. 2010. The lessons learned handbook: Practical knowledge-based approach to learning from experience. Chandos Publishing Ltd. Oxford.

Muntaner, J., Roselló, M. y De La Iglesia, B. 2016. Buenas prácticas en educación inclusiva. Educatio Siglo XXI, 34(1), pp.31-50.

Nonaka, I. 1991. The knowledge-creating company. Harvard Business Review, July-August 2007. Boston.

Nonaka, I. y Takeuchi H. 1995. The knowledge-creating company: How Japanese companies create the dynamics of innovation. Oxford University Press. New York.

Pérez, J. 2008. Definición de conocimiento. Recuperado de: https://definicion.de/conocimiento//. Fecha de consulta: 6 de marzo de 2018.

Rigby, D. 2011. Management Tools 2011. An Executive's Guide, p. 76. Recuperado de: http://www.bain.com/ Images/Bain_Management_Tools_2011.pdf. Fecha de consulta: 13 de enero de 2017.

Sánchez, M. F. 2016. Gestión del Conocimiento. Instituto Tecnológico Nacional de México, 1-17.

Santiso, C., 2002. Education for democratic governance: review of learning programmes. Org. Social Sciences. UNESCO.

Tan, H. C., Carrillo, P., Anumba, C., Kamara, J. M., Bouchlaghem, D. y Udeaja, C. 2006. Live capture and reuse of project knowledge in construction organizations. Knowledge Management Research and Practice, 4(2), 149-161.

UNS. 2017. Guía para la documentación de proyectos de software. Universidad Nacional del Sur. Argentina.

Van Wart, M., Roman, A., Wang, X. y Liu, C. 2017. Integrating ICT adoption issues into (e-)leadership theory. Telematics and Informatics, 34 (2017) 527-537.

Visser, J. 2017. Building software Teams. Ten best practices for effective software development. O'Reilly Media, Inc. Sebastopol.

Weber, R., Aha, D. W. y Becerra-Fernandez, I. 2001. Intelligent lessons learned systems. Journal of Expert Systems Research \& Applications, 20(1), 17-34

Wiig, K. 1993. Knowledge management foundations: Thinking about thinking. How people and organizations create, represent and use knowledge. Schema Press. Arlington, TX. 